\title{
Nomenclatura en química orgánica
}

\section{Beatriz Giner ${ }^{a}$, Laura Lomba ${ }^{b}$, Diego Ballestero ${ }^{c}, \mathbf{M}^{a}$ Pilar Ribate $^{d}$}

${ }^{a}$ Facultad de Ciencias de la Salud, Universidad San Jorge, Campus Universitario Villanueva de

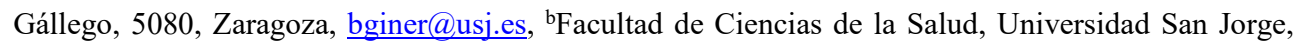

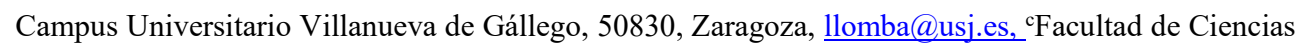
de la Salud, Universidad San Jorge, Campus Universitario Villanueva de Gállego, 50830, Zaragoza, dballestero@usj.es, ${ }^{\mathrm{d}} \mathrm{Facultad}$ de Ciencias de la Salud, Universidad San Jorge, Campus Universitario Villanueva de Gállego, 50830, Zaragoza mpribate@usj.es.

\begin{abstract}
This work shows various activities carried out during the 2018-2019 academic year to improve the competences in "Nomenclature in organic chemistry" of the students of the degree in Pharmacy of the Universidad San Jorge. Activities are linked to the subjects "Organic Chemistry" and "Biochemistry and Molecular Biology I" corresponding to two different courses. The activities have been based on student-centered learning with the aim of facilitating the learning of organic nomenclature and improving the attitude of students regarding these contents. The activities carried out by the students include the elaboration of a book of activities to practice organic nomenclature and the design and elaboration of resources that can be used both for study and for support in the examination of this part of the subject.
\end{abstract}

Keywords: organic chemistry, nomenclature, transversal, innovation, methodology.

\section{Resumen \\ En este trabajo se muestran diversas actividades realizadas en durante el curso 2018-2019 para mejorar las competencias en "Nomenclatura en química orgánica" de los alumnos del grado en Farmacia de la Universidad San Jorge. Se han realizado actividades en las asignaturas "Química Orgánica" y Bioquímica y Biología Molecular I correspondientes a dos cursos diferentes. Las actividades se han basado en el aprendizaje centrado en el alumno con el objetivo de facilitar el aprendizaje de la nomenclatura orgánica y mejorar la actitud de los alumnos respecto a estos contenidos. Las actividades realizadas por los alumnos incluyen la elaboración de un libro de actividades para practicar la nomenclatura orgánica y el diseño y elaboración de recursos que pueden ser utilizados tanto para el estudio como de apoyo en el examen de esta parte de la materia.}

Palabras clave: química orgánica, nomenclatura, transversal, innovación, metodología. 


\section{Introducción}

En el grado en Farmacia de la Universidad San Jorge se imparten diversas asignaturas relacionadas con la Química tales como Química Inorgánica, Química Orgánica, Fisicoquímica, Química Farmacéutica o Bioquímica y Biología Molecular, entre otras. Para ser competente en estas asignaturas, se requiere de una formación previa en nomenclatura química. La nomenclatura, definida como la parte de la Química que clasifica y nombra las sustancias químicas, es una herramienta fundamental para el aprendizaje, aplicación y dominio de estas materias. La Intrenational Union of Pure and Applied Chemistry, IUPAC, es la autoridad internacional en nomenclatura y terminología química, que ha desarrollado un lenguaje común para todos los químicos, así como la estandarización de procesos y procedimientos.

Gracias a la nomenclatura química somos capaces de nombrar cualquiera de las más de 147 millones de sustancias orgánicas e inorgánicas descritas en la literatura desde principios de 1800 (https://www.cas.org/about/cas-content, acceso 15 de marzo de 2019). Además, la nomenclatura asigna los átomos que forman la molécula y su disposición de manera que permite al químico predecir las propiedades, tanto químicas como físicas de las sustancias químicas. Es evidente entonces que el aprendizaje en química requiere conocer lenguaje específico y saber aplicar una serie de reglas, de modo que se pueda deducir el nombre y estructura de cualquier sustancia química.

Es bien conocido, que existen diversas teorías de enseñanza que permiten explicar cómo se produce el aprendizaje. Una de ellas es el aprendizaje por descubrimiento y aprendizaje centrado en el alumno (Ontoria, 2006; Investigaciones cualitativas en ciencia y tecnología, 2017). Según esta teoría, el profesor es un guía del aprendizaje mientras que el alumno descubre el conocimiento por sí mismo. Existen varias experiencias previas en las que los alumnos son protagonistas de su aprendizaje y preparan íntegramente sesiones en las que muestran lo aprendido al resto de sus compañeros, incluyendo actividades y ejercicios para realizar en el aula preparados por ellos mismos (Álvarez, 2008) así como elaboración de material docente por alumnos. También se ha referenciado previamente el uso de cuestionarios y juegos para favorecer el aprendizaje de la nomenclatura orgánica de manera lúdica y positiva (Gordon, 1983; Rodríguez, 2017).

El proyecto que se presenta a continuación está basado en el aprendizaje por descubrimiento y ha permitido a los alumnos de primer y segundo curso del grado en Farmacia de la Universidad San Jorge, aprender nomenclatura orgánica por sí mismos y reforzar lo aprendido a lo largo del tiempo, transversalmente en diversas asignaturas del grado. Las actividades realizadas en este proyecto han sido 
planificadas con el objetivo de facilitar el aprendizaje de la nomenclatura orgánica y mejorar la actitud de los alumnos respecto a la misma. De esta forma, se establece una base para un mejor desarrollo de las asignaturas del grado en Farmacia que hacen uso de la Química Orgánica como base del conocimiento del resto de materias.

\section{Objetivos}

El objetivo principal del proyecto es facilitar el aprendizaje de la nomenclatura orgánica. Para ello, se ha elaborado un dossier que contiene diversas actividades encaminadas a mejorar la disposición de los alumnos hacia el aprendizaje de la misma y aumentar la seguridad de los alumnos de cara a la realización del examen final de esta parte de la asignatura.

Como objetivos secundarios destacamos los siguientes:

- Aprender a dibujar moléculas orgánicas correctamente utilizando diversos programas informáticos.

- Elaborar un libro de actividades para practicar la nomenclatura orgánica.

\section{Desarrollo de la innovación}

La actividad que se presenta ha consistido en la generación de un dossier de actividades para favorecer el aprendizaje de nomenclatura orgánica. En esta actividad han participado alumnos de las asignaturas Química Orgánica y Bioquímica y Biología Molecular I del grado en Farmacia de la Universidad San Jorge. Las actividades han sido diseñadas y ejecutadas tanto por los profesores de la asignatura como por los propios alumnos, de modo que el proceso de diseño y realización ha servido como aprendizaje de la materia.

En la figura 1, se muestran la secuencia de actividades, por asignatura y curso. 


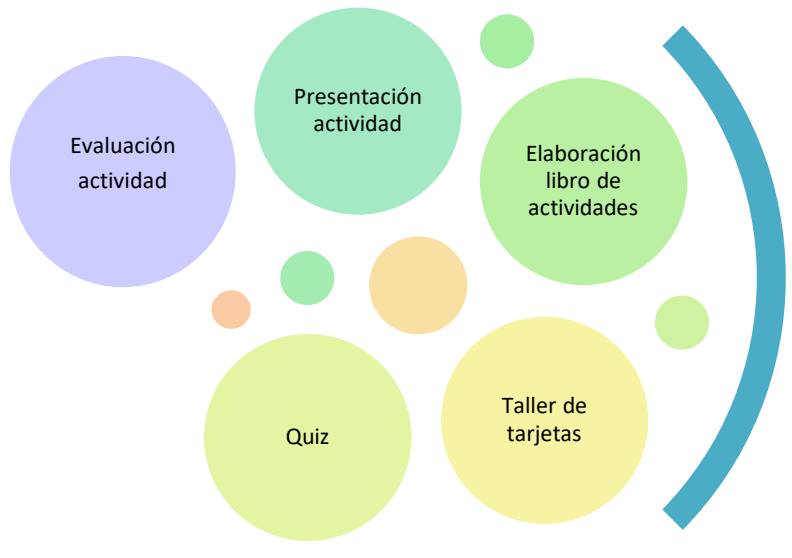

Año 1

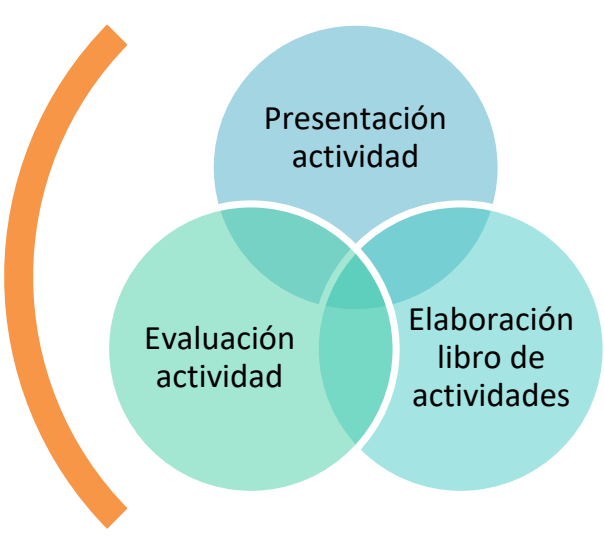

Año 2

Química Orgánica

Bioquímica y Biología Molecular 1

Figura 1: Planificación de la actividad de innovación desarrollada.

Las actividades que se han llevado a cabo en la asignatura de Química Orgánica de primer curso del grado en Farmacia de la Universidad San Jorge son las siguientes:

a) Presentación de la actividad. Explicación del desarrollo y método de evaluación, entrega de recursos y calendario.

b) Elaboración de libro de actividades para practicar nomenclatura orgánica. Cada alumno ha ideado 5 moléculas orgánicas, conteniendo diversos grupos funcionales y las nombró adecuadamente. Se ha utilizado el programa informático Chemsketch para representar las moléculas. Los profesores de la asignatura han utilizado esa información para elaborar un libro de actividades, que se podrá utilizar en cursos posteriores como recursos de la asignatura. Se ha pretendido implicar así a todos los alumnos y mejorar su actitud, puesto que al completar esta actividad serán autores de un libro.

c) Taller de tarjetas con información clave. Por grupos, los alumnos han diseñado unas tarjetas con información importante para nombrar las moléculas orgánicas. Se ha seleccionado la mejor tarjeta, se ha impreso y ha sido utilizada tanto como material de estudio como material de apoyo en el examen. De este modo, hemos intentado rebajar la ansiedad que produce el examen de nomenclatura $\mathrm{y}$ hemos asegurado entusiasmo y buen hacer en esta actividad, puesto que el resultado de la misma es una excelente ayuda en los exámenes. 
d) Quiz. Por grupos, los alumnos han preparado un cuestionario con 10 preguntas (nomenclatura y formulación). Estos cuestionarios, preparados por los propios alumnos, han sido utilizados como material de preparación al estudio y control de aprendizaje. Se han realizado 3 pruebas previas al examen final de nomenclatura durante las sesiones de teoría utilizando las pruebas preparadas por los alumnos, que han podido así comprobar el avance del aprendizaje, utilizando sus propios recursos.

Todas las actividades se han llevado a cabo durante las 4 primeras semanas del curso 2018-2019 (febrero 2019), ya que la nomenclatura se ha de trabajar al principio del curso, base del resto de la asignatura.

Las actividades que se han llevado a cabo en la asignatura de Bioquímica y Biología Molecular I de segundo curso del grado en Farmacia de la Universidad San Jorge son las siguientes:

a) Presentación de la actividad. Explicación del desarrollo y método de evaluación, entrega de recursos y calendario.

b) Elaboración de libro de actividades para practicar nomenclatura orgánica. Con intención de continuar practicando el uso de los recursos informáticos y la nomenclatura orgánica, se ha destinado un capítulo del libro de actividades, descrito previamente, a la nomenclatura de biomoléculas. En este caso el trabajo se ha realizado por parejas; cada una de ellas ha elegido 5 biomoléculas, conteniendo diversos grupos funcionales y las ha nombrado adecuadamente. Del mismo modo que en el apartado anterior, se han representado las moléculas con Chemsketch. Estas actividades se incorporarán al libro de actividades en nomenclatura orgánica. Esta actividad se ha llevado a cabo durante las 2 últimas semanas del primer semestre del curso 2018-2019 (enero 2019).

\section{Resultados}

En esta actividad han participado 49 alumnos pertenecientes a 2 grupos de Química Orgánica y 33 alumnos pertenecientes a la asignatura Bioquímica y Biología Molecular I.

Como resultado de la actividad "Elaboración de libro de actividades para practicar nomenclatura orgánica", se han obtenido aproximadamente 400 moléculas orgánicas, nombradas y representadas mediante Chemsketch que serán la base del libro de actividades que se editará en los próximos meses.

Tras la actividad Quiz, se han obtenido 15 documentos conteniendo cada uno de ellos, 20 moléculas orgánicas (10 nomenclatura y 10 formulación). Este material se 
ha utilizado para elaborar las 3 pruebas de control realizadas en las semanas previas al examen final de nomenclatura.

Como resultado del "Taller de tarjetas con información clave", se han obtenido un total de 16 tarjetas. Se adjunta la tarjeta seleccionada que fue utilizada en el examen final de nomenclatura (figura 2).

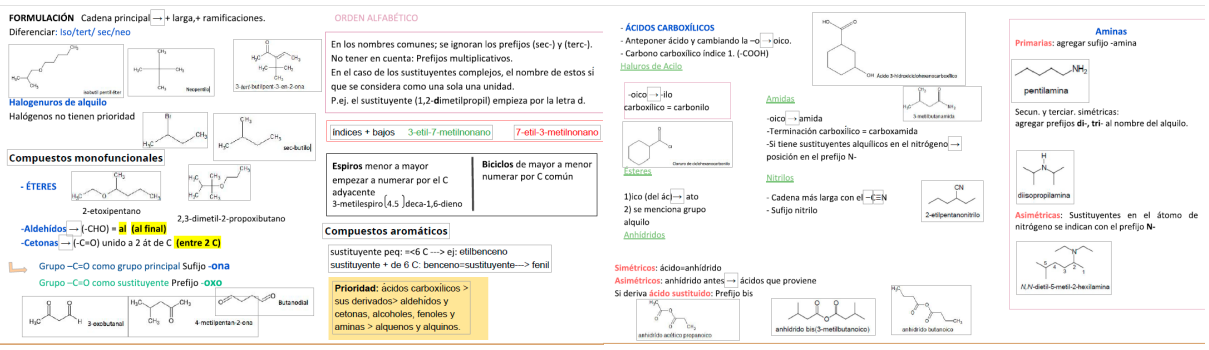

Figura 2. Tarjeta seleccionada de la actividad "Taller de tarjetas con información clave".

A continuación, se describen los resultados obtenidos en la prueba final de nomenclatura realizada el 15 de marzo de 2019. De los 48 alumnos matriculados en la asignatura Química Orgánica, se presentaron al examen 41 alumnos. El 66\% de los alumnos obtuvo una calificación "APTO", mientras que el 34\% restante no superó la prueba. En la figura 3 se muestra una comparativa de los resultados obtenidos desde el curso 2015-2016. Destacar que los resultados del curso 20182019 se corresponde con una única prueba, mientras que en los cursos anteriores, los resultados son acumulados de tres pruebas (la prueba de marzo, equivalente a la realizada el 15 de marzo de 2019 y dos recuperaciones más, correspondientes a las convocatorias de junio y julio de los respectivos cursos académicos). Es por tanto esperable, que los resultados de este curso 2018-2019 mejoren con respecto a los de años anteriores, ya que faltan todavía por incluir las dos recuperaciones en las convocatorias de junio y julio.

\section{Resultados obtenidos en el examen de nomenclatura}

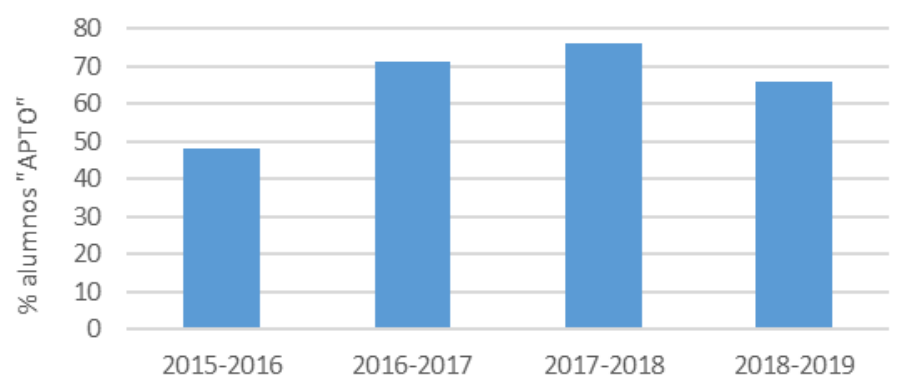

Figura 3. Resultados obtenidos en la prueba final de nomenclatura orgánica desde el curso 20152016. 
La evaluación de la actividad realizada por los alumnos de la asignatura de Bioquímica y Biología molecular I estaba englobada en el apartado de trabajo en grupo. En este caso todos los alumnos entregaron la actividad correctamente desarrollada y en plazo a excepción de dos grupos que no utilizaron el programa indicado en las bases de la actividad (Chemsketch) y por lo tanto dicha actividad se evaluó con una calificación "No Apto" para ambos. En la figura 4 aparecen varias de las biomoléculas seleccionadas por estos alumnos y que se aportarán al libro de actividades.
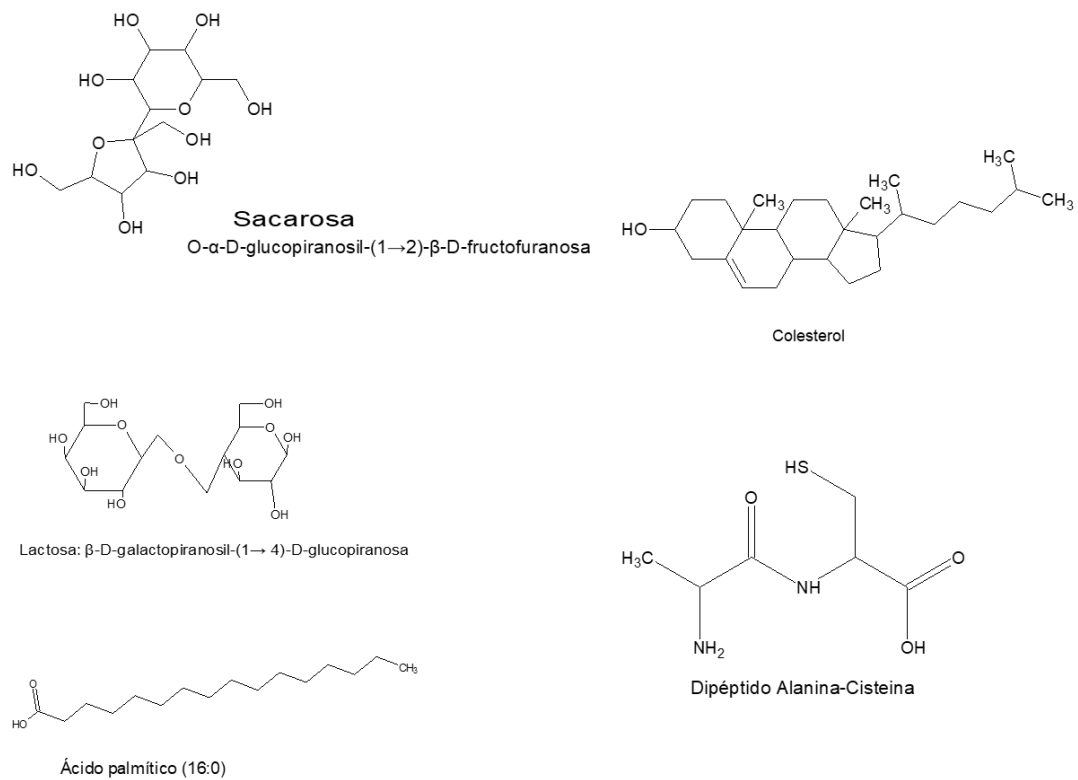

Dipéptido Alanina-Cisteina

Figura 4. Ejemplos de las biomoléculas seleccionadas por los alumnos de la asignatura de Bioquímica y Biología molecular I.

Además, se ha realizado una evaluación cuantitativa de la percepción de los estudiantes acerca de las actividades propuestas. Esta evaluación se ha realizado mediante una encuesta en la que se les preguntaba a los alumnos sobre la utilidad del proyecto en general. Asimismo, se preguntaba específicamente por la utilidad de cada una de las actividades realizadas. También se incluían preguntas relacionadas con la mejora del aprendizaje a largo plazo.

En la figura 5 se muestran los resultados generales obtenidos en esta evaluacón. Sólo el $6 \%$ de los estudiantes han percibido la actividad como poco útil. La mayoría de los alumnos han concluido que el proyecto propuesto ha sido útil o muy útil para su formación. Asimismo, ninguno de los alumnos encuestados ha 
declarado que no han mejorado sus competencias en nomenclatura, mientras que el $94 \%$ opina que ha mejorado gracias a esta actividad.
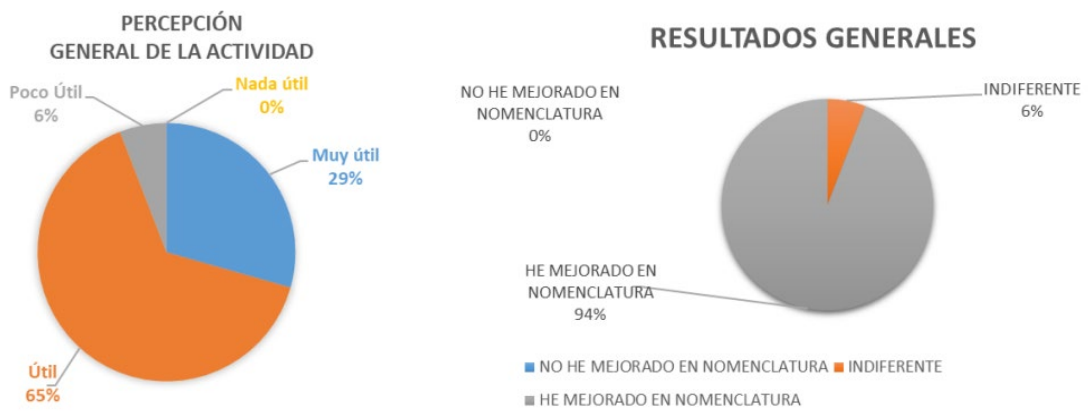

Figura 5. Percepción general de la actividad por los estudiantes.

Disgregando los resultados por cada una de las tres actividades realizadas, encontramos que la actividad "Libro" es la que menos útil les ha parecido a los alumnos, mientras que la actividad "Taller de tarjetas" es la que les ha resultado más provechosa (figura 6).

UTILIDAD DE LAS DIFERENTES ACTIVIDADES

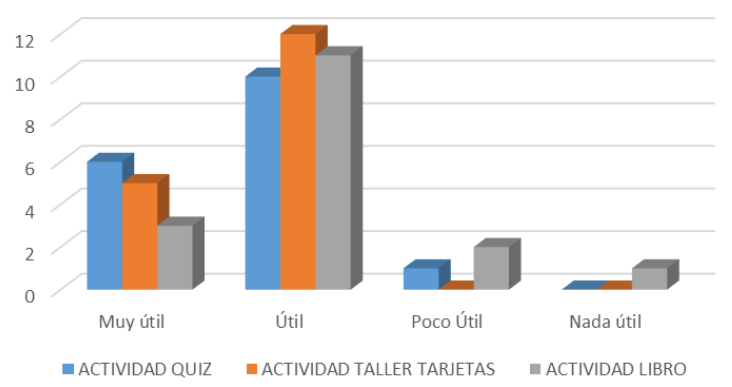

Figura 6. Percepción disgregada de las diferentes actividades por los estudiantes.

Finalmente, en la figura 7 se muestra la percepción de futuro cuando se les pregunta a los alumnos por la utilidad del proyecto en asignaturas de otros cursos y en su futura profesión. 


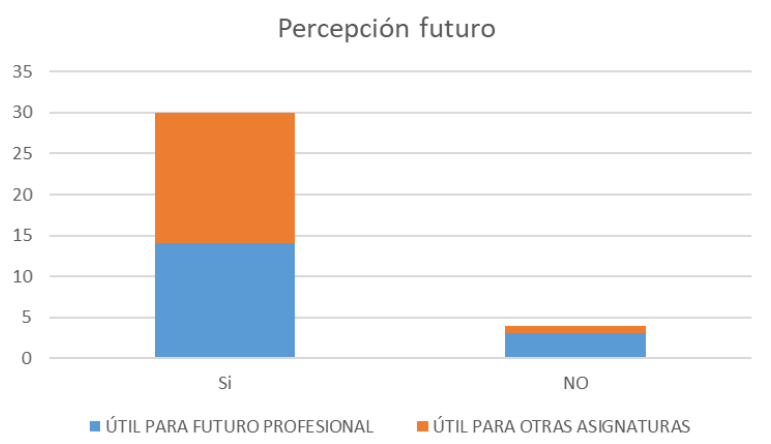

Figura 7. Percepciónde los alumnos acerca de la utilidad de las actividades para el futuro..

\section{Conclusiones}

Este proyecto está basado en el aprendizaje centrado en el alumno, de modo que es él mismo, el que tiene que desarrollar las actividades que sirven de práctica tanto para él como para el resto de sus compañeros. Asimismo, mediante la actividad "taller de tarjetas" se ha trasladado al alumno la responsabilidad de elaborar un buen material que puede utilizar en los exámenes. Se ha apreciado una notable mejora en cuanto a la actitud del alumno al respecto del aprendizaje de esta parte. Disponer de material de apoyo en el examen les ha proporcionado seguridad y tranquilidad. Destacar que la información recogida en la tarjeta de apoyo era meramente mecanística, si bien, se necesitaba de un trabajo de profundización en nomenclatura orgánica para poder aplicar las normas allí descritas con soltura. En el caso de los alumnos de la asignatura de Bioquímica y Biología molecular I, el desarrollo de esta actividad les ha permitido recordar y reforzar los conocimientos de nomenclatura orgánica adquiridos en el curso anterior. Lo que ayuda a evitar la compartimentalización habitual que realizan los alumnos sobre las distintas asignaturas del grado.

Varias de las actividades planteadas se trabajaron en grupo, de modo que se favorece también la adquisición de esta competencia.

Por otro lado, se utilizan diversos recursos informáticos, que han potenciado el desarrollo en este ámbito y que, además, se prolongan en el tiempo, puesto que el proyecto realizado cubre asignaturas pertenecientes a dos cursos.

Fruto de este proyecto es la colección de un conjunto de materiales y recursos, que estarán disponibles en cursos posteriores y también serán de utilidad para cualquier curso de Química Orgánica o Bioquímica, tanto de la USJ como de otros centros de enseñanza. 
Los alumnos han acogido el proyecto con buena disposición y los resultados muestran que la mayoría de ellos han percibido la utilidad de las actividades propuestas.

\section{Referencias}

ÁlVAREZ, F. RODRÍGUEZ-PÉREZ, J. R. SANZ-ABLANEDO, E. FERNÁNDEZMARTÍNEZ. M (2008) “Aprender enseñando: elaboración de materiales didácticos que facilitan el aprendizaje autónomo" en Formación universitaria. 2008, Vol 1(16), 19-28.

GORDON F. (1983) "Organic nomenclature: Making it a more exciting teaching and learning experience” en J. Chem. Educ., 1983, Vol 60 (7), 553.

ONTORIA, A. (2006) Aprendizaje centrado en el alumno. Metodología para una escuela abierta. Madrid: Narcea S.A de ediciones.

https://www.cas.org/about/cas-content, acceso 15 de marzo de 2019.

Investigaciones cualitativas en ciencia y tecnología (2017) VI congreso internacional de investigación cualitativa en ciencia y tecnología.

RODRIGUEZ, F. SANTIAGO, R. (2017) Gamificación: Cómo motivar a tu alumnado y mejorar el clima en el aula (Innovación educativa). Digital-Text. 